\title{
On the Computation of Steady Hopper Flows III: Model Comparisons
}

\author{
Pierre A. Gremaud ${ }^{\mathrm{a}, 1}$, John V. Matthews ${ }^{\mathrm{b}, 2}$, \\ David G. Schaeffer ${ }^{\mathrm{c}, 2}$, \\ ${ }^{a}$ Department of Mathematics and Center for Research in Scientific Computation, \\ North Carolina State University, Raleigh, NC 27695-8205, USA \\ ${ }^{\mathrm{b}}$ Department of Mathematics, University of Tennessee at Chattanooga, TN \\ 37403-2504, USA \\ ${ }^{\mathrm{c}}$ Department of Mathematics and Center for Nonlinear and Complex Systems, \\ Duke University, Durham, NC 27708-0320
}

\begin{abstract}
Gravity flows of granular materials through hoppers are considered. For hoppers shaped as general nonaxisymmetric cones, i.e., "pyramids", the flow inherits some simplified features from the geometry: similarity solutions can be constructed. Using two different plasticity laws, namely Matsuoka-Nakai and von Mises, those solutions are obtained by solving first order nonlinear partial differential algebraic systems for stresses, velocities, and a plasticity function.

A pseudospectral discretization is applied to both models and the resulting flow fields are examined. Some similarities are found, but important differences appear, especially with regard to velocities near the wall and normal wall stresses. Preliminary comparisons with recent experiments [11] based on the present results indicate that for slow granular flows the lesser known Matsuoka-Nakai plasticity law yields better results than more common models based on a von Mises criterion.
\end{abstract}

Key words: granular, similarity, spectral, plasticity

Email addresses: gremaud@ncsu.edu (Pierre A. Gremaud), Matt-Matthews@utc.edu (John V. Matthews), dgs@math.duke.edu (David G. Schaeffer).

URLs: http://www.ncsu.edu/math/ gremaud (Pierre A. Gremaud), http://www.utc.edu/Faculty/Matt-Matthews/ (John V. Matthews), http://www. math. duke.edu/ dgs (David G. Schaeffer).

1 Partially supported by the National Science Foundation (NSF) through grants DMS-0204578, DMS-0244488 and DMS-0410561

2 Partially supported by the National Science Foundation (NSF) through grant 


\section{Introduction}

This is the third in a series of papers on the mechanics of a granular material under the influence of gravity flowing through converging hoppers. Calculations of granular flows are typically subject to severe geometric restrictions. Jenike [6] in the late 1950's was the first to realize that similarity solutions to the equations of motion can easily be constructed in hoppers of very simple geometry. Working exclusively in cones $^{3}$

$$
\left\{(r, \theta, \phi): 0<r<\infty, 0 \leq \theta<\theta_{w}\right\}, \quad\left(\theta_{w}=\text { constant }\right)
$$

Jenike constructed similarity solutions that are still the bedrock of most engineering studies in this field. Those solutions inherit both the self-similarity of the domain ( $r$ scales out) and its axisymmetry (the solutions only depend on $\theta$ and solve a system of ODEs). In [4], the authors generalized this approach to "pyramids", i.e., self-similar but not necessarily axisymmetric domains such as

$$
\{(r, \theta, \phi): 0<r<\infty, 0 \leq \theta<\mathcal{C}(\phi)\}
$$

where $\mathcal{C}$ is a given piecewise smooth $2 \pi$-periodic function describing the boundary of the cross section of the hopper. While the corresponding solutions are of course still self-similar, their structure is much more involved. Those previous results all use plasticity models based on a von Mises condition [10] or a simplified Mohr-Coulomb condition [8].

Another model was proposed by Matsuoka and Nakai in [7] and recently studied in [3]. In the present paper, the approach from [4] is applied to MatsuokaNakai materials for comparison with von Mises ones. In Section 2, both models are recalled. Radial solutions in right conical domains for the Matsuoka-Nakai model are constructed (for the first time) in Section 3. The two models are applied to pyramidal domains (2) in Section 4. Numerical results are presented Section 5 while concluding remarks are offered in Section 6.

Our work is motivated by preliminary comparisons with experimental values $[1,11]$ that seem to indicate a much better match with the lesser known Matsuoka-Nakai condition than with the von Mises one.

DMS-0244492

3 Spherical coordinates are used exclusively in this paper: $(r, \theta, \phi)$ are the usual spherical coordinates, $\phi$ being the azimuthal angle. In (1), $\theta_{w}$ is the half opening angle of the cone. 


\section{Plasticity models}

The unknowns are the 3 -component velocity vector $v$, the $3 \times 3$ symmetric stress tensor $T$, and a scalar plasticity coefficient $\lambda$. The density $\rho$ is assumed constant. For slow flows, inertia can be neglected in Newton's second law and thus balance of forces reads

$$
\nabla \cdot T=\rho g
$$

where $g$ is the (vector) acceleration of gravity. In addition to (3) both models consider two constitutive laws: a flow rule and a plasticity law.

\section{von Mises model}

$$
\begin{aligned}
V & =\lambda \operatorname{dev} T, \\
|\operatorname{dev} T|^{2} & =2 s^{2}(\operatorname{tr} T / 3)^{2} .
\end{aligned}
$$

\section{Matsuoka-Nakai model}

$$
\begin{aligned}
V & =-\lambda \operatorname{dev} T^{-1}, \\
(\operatorname{tr} T)\left(\operatorname{tr} T^{-1}\right) & =9\left(1+\mu^{2}\right) .
\end{aligned}
$$

In the above equations, $V=-1 / 2\left(\nabla v+\nabla v^{T}\right)$ is the strain rate tensor (note the sign convention) while $\operatorname{dev} T=T-\frac{1}{3}(\operatorname{tr} T) \mathrm{I}$ is the deviatoric part of the stress tensor. Further, $|\cdot|$ denotes the Frobenius norm $|T|^{2}=\sum_{i, j=1}^{3} T_{i j}^{2}$. The material parameters $s$ and $\mu$ are such that $s=\sin \delta$ and $\mu=\tan \delta, \delta$ being the angle of internal friction of the material under consideration (see [8]).

The properties of the yield surfaces defined by (5) and (7) are well known $[2,3,10]$ and can be easily established in principal stress space, i.e., in terms of the eigenvalues $\left\{\sigma_{i}\right\}_{i=1}^{3}$ of the stress tensor $T$. For granular materials, the stress tensor $T$ measures compressive stresses, i.e., the principal stresses are positive. The von Mises yield surface (5) is a cone with two nappes. One nappe is contained in the physically relevant positive octant $\left\{\sigma_{i}>0, i=1,2,3\right\}$ if and only if $\delta<60^{\circ}[10]$. The structure of the Matsuoka-Nakai yield surface is more complicated, but again, a cone of one nappe is contained in the positive octant (regardless of $\delta$ ), see [2] for details on that case.

Inside a container, the flow is subject to boundary conditions. Wall impenetrability reads

$$
v_{N}=0,
$$


where $v_{N}=v \cdot N$ is the normal velocity, $N$ being the unit interior normal. Two additional boundary conditions come from Coulomb's law of sliding friction. The surface traction $\tau$-i.e., the force exerted by the wall on the materialis given by $\tau_{i}=\sum_{j=1}^{3} T_{i j} N_{j}$. If the vector $\tau$ has normal component $\tau_{N}$ and tangential component $\tau_{T}=\tau-\tau_{N} N$, then

$$
\tau_{T}=-\mu_{w} \tau_{N}(v /|v|),
$$

where $\mu_{w}$ is the coefficient of sliding friction between the wall and the material.

\section{Radial flows}

The first hopper geometry we consider is that of a regular vertical cone of equation (1). As first noticed by Jenike [6], the invariance of the domain (1) under the transformation $r \mapsto c r, c>0$, leads to the existence of similarity solutions. For Matsuoka-Nakai, these solutions scale as

$$
T_{i j}(r, \theta, \phi)=r \mathcal{T}_{i j}(\theta, \phi), v_{i}(r, \theta, \phi)=\frac{1}{r^{2}} \mathcal{V}_{i}(\theta, \phi), \lambda(r, \theta, \phi)=\frac{1}{r} \mathcal{L}(\theta, \phi),
$$

where $T_{i j}$ and $v_{i}$ are any component of the stress tensor and velocity vector respectively. For von Mises, the scaling for $\lambda$ differs. See [4].

We now derive the equations governing the flow in the domain (1) for the Matsuoka-Nakai model. The corresponding equations for the von Mises case have been extensively studied elsewhere $[6,8,10]$. By axisymmetry, the (scaled) stress tensor and velocity vector respectively simplify to

$$
\mathcal{T}=\left[\begin{array}{ccc}
\mathcal{T}_{r r} & \mathcal{T}_{r \theta} & 0 \\
\mathcal{T}_{r \theta} & \mathcal{T}_{\theta \theta} & 0 \\
0 & 0 & \mathcal{T}_{\phi \phi}
\end{array}\right] \quad \text { and } \quad \mathcal{V}=\left[\begin{array}{c}
\mathcal{V}_{r} \\
0 \\
0
\end{array}\right]
$$

Under the above assumptions, the grains follow radial lines; the corresponding solutions are referred to as radial solutions. Assuming the stress and velocity field inherit the axisymmetric character of the domain, none of the above variables depends on $\phi$. The problem is thus reduced to finding the six unknowns $\mathcal{T}_{r r}, \mathcal{T}_{r \theta}, \mathcal{T}_{\theta \theta}, \mathcal{T}_{\phi \phi}, \mathcal{V}_{r}$ and $\mathcal{L}(\mathcal{L}$ is eliminated below $)$

Conservation of momentum (3) yields two nontrivial scalar relations, another equation comes from the yield condition (7) while two come from the flow rule $(6)$ after elimination of $\lambda$ (and thus $\mathcal{L}$ ). Under the above assumptions, the 
$\theta \theta$ - and $\phi \phi$-components of the strain rate tensor are equal. The flow rule (6) implies that $\left(\operatorname{dev} T^{-1}\right)_{\theta \theta}=\left(\operatorname{dev} T^{-1}\right)_{\phi \phi}$, or equivalently

$$
\mathcal{T}_{r r} \mathcal{T}_{\phi \phi}=\mathcal{T}_{r r} \mathcal{T}_{\theta \theta}-\mathcal{T}_{r \theta}^{2}, \quad \text { in }\left[0, \theta_{w}\right]
$$

The yield condition (7) together with the above relation then leads to

$$
\left(\mathcal{T}_{r r}+\mathcal{T}_{\theta \theta}+\mathcal{T}_{\phi \phi}\right)\left(2 \mathcal{T}_{r r}+\mathcal{T}_{\theta \theta}\right)=9\left(1+\mu^{2}\right) \mathcal{T}_{r r} \mathcal{T}_{\phi \phi}, \quad \text { in }\left[0, \theta_{w}\right]
$$

The two equations contributed by conservation of momentum are as follows

$$
\begin{aligned}
\frac{d}{d \theta} \mathcal{T}_{r \theta}+3 \mathcal{T}_{r r}+\cot \theta \mathcal{T}_{r \theta}-\mathcal{T}_{\theta \theta}-\mathcal{T}_{\phi \phi} & =-\rho g \cos \theta, \quad \\
\frac{d}{d \theta} \mathcal{T}_{\theta \theta}+4 \mathcal{T}_{r \theta}+\cot \theta\left(\mathcal{T}_{\theta \theta}-\mathcal{T}_{\phi \phi}\right) & =\rho g \sin \theta, \quad \text { in }\left(0, \theta_{w}\right)
\end{aligned}
$$

Equations (12-15) form an independent system for the stress unknowns $\mathcal{T}_{r r}$, $\mathcal{T}_{r \theta}, \mathcal{T}_{\theta \theta}, \mathcal{T}_{\phi \phi}$. Equation (13) can be rewritten

$$
\left[\begin{array}{lll}
\mathcal{T}_{r r} & \mathcal{T}_{\theta \theta} & \mathcal{T}_{\phi \phi}
\end{array}\right]\left[\begin{array}{ccc}
2 & 3 / 2 & 1-\frac{9}{2}\left(1+\mu^{2}\right) \\
3 / 2 & 1 & 1 / 2 \\
1-\frac{9}{2}\left(1+\mu^{2}\right) & 1 / 2 & 0
\end{array}\right]\left[\begin{array}{l}
\mathcal{T}_{r r} \\
\mathcal{T}_{\theta \theta} \\
\mathcal{T}_{\phi \phi}
\end{array}\right]=0
$$

The matrix appearing in the previous relation has full rank, its determinant is equal to $-18-\frac{153}{4} \mu^{2}-\frac{81}{4} \mu^{4}<0$, and consequently its eigenvalues have different signs. Therefore (13) defines a real quadratic cone.

The second equation resulting from the flow rule (6) can be written

$$
\frac{d}{d \theta} \mathcal{V}_{r}=-\frac{6 \mathcal{T}_{r \theta}}{\mathcal{T}_{r r}-T_{\theta \theta}} \mathcal{V}_{r}, \quad \text { in }\left(0, \theta_{w}\right)
$$

The complete system (12-16) which consists of two algebraic constraints and three ODEs is closed by three boundary conditions

$$
\begin{aligned}
\mathcal{T}_{r \theta}(0) & =0, \\
-\mathcal{T}_{r \theta}\left(\theta_{w}\right) & =\mu_{w} \mathcal{T}_{\theta \theta}\left(\theta_{w}\right), \\
\mathcal{V}_{r}(0) & =-1 .
\end{aligned}
$$

Condition (17) corresponds to $\mathcal{T}_{r \theta}$ being odd in this geometry. Relation (18) directly follows from Coulomb's law of sliding friction (9). Finally, (19) is a 

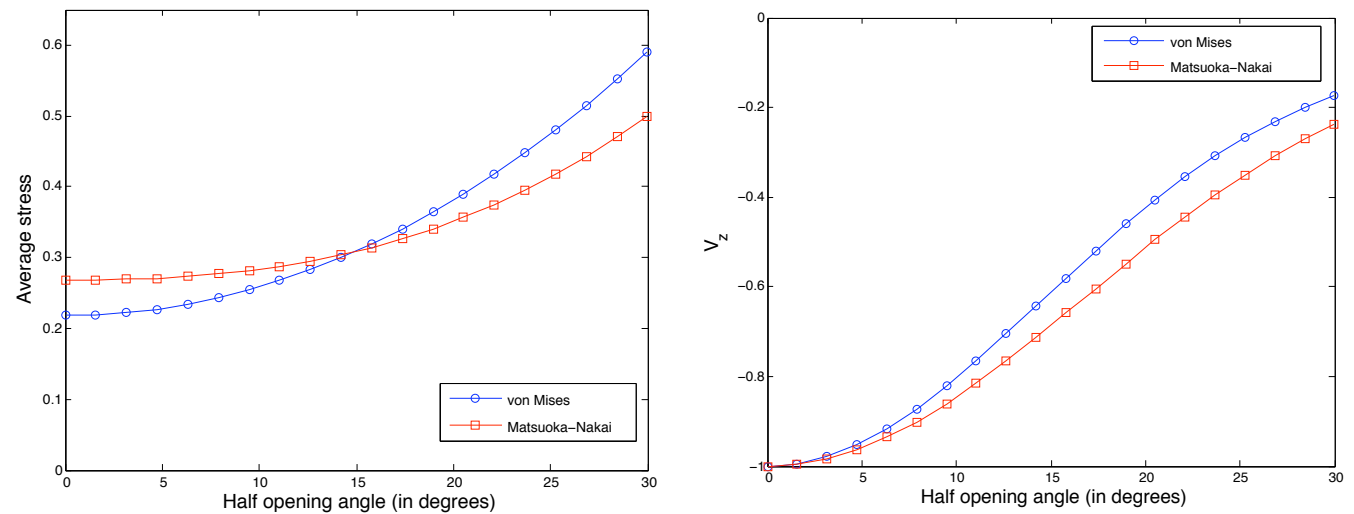

Fig. 1. Radial solution flow for both models; left: comparison of average stress $\left(\mathcal{T}_{r r}+\mathcal{T}_{\theta \theta}+\mathcal{T}_{\phi \phi}\right) / 3$, right: comparison of vertical velocity $\mathcal{V}_{z}$. Material parameters: angle of internal friction $\delta=30^{\circ}$, half opening angle $\theta_{w}=30^{\circ}$, coefficient of wall friction $\mu_{w}=0.3$.

normalization condition, as (16) only defines $\mathcal{V}_{r}$ up to a positive multiplicative constant.

To solve the above system numerically, the interval $\left[0, \theta_{w}\right]$ is divided into $N$ cells of equal size $\Delta \theta=\theta_{w} / N$. The numerical unknowns are approximate values at the nodes $\theta^{i}, i=0, \ldots, N$, i.e., $\mathcal{T}_{r r}^{i}, \mathcal{T}_{r \theta}^{i}, \mathcal{T}_{\theta \theta}^{i}, \mathcal{T}_{\phi \phi}^{i}$ and $\mathcal{V}_{r}^{i}$. Those unknowns are determined as follows

- the $\mathcal{T}_{\phi \phi}^{i}$ 's are eliminated through (12),

- equations (14-16) are discretized by the midpoint rule, i.e., at $\theta^{i+1 / 2}=$ $\left(\theta^{i}+\theta^{i+1}\right) / 2, i=0, \ldots, N-1$,

- equation (13) is enforced at the nodes $\theta_{i}, i=0, \ldots, N$,

- the boundary conditions (17-19) are imposed.

This results in a system of $5(N+1)$ nonlinear equations ${ }^{4}$. The velocity can be computed in a postprocessing step as the stress equations do not depend on it.

Radial solutions are shown in Figure 1. While the two models differ somewhat in this geometry, much greater differences become apparent in nonaxisymmetric hoppers.

$\overline{4}$ The current nonlinear solver uses a trust region dogleg method [9] with finite difference Jacobians. 


\section{Flows in pyramidal domains}

The geometry is now generalized from right regular cones (1) to "pyramidal domains" (2). While the scaling argument leading to (10) is still valid, the loss of axisymmetry prevents the simplifications that lead to (11). Consequently, all ten unknowns $\mathcal{T}_{r r}, \mathcal{T}_{r \theta}, \mathcal{T}_{\theta \theta}, \mathcal{T}_{r \phi}, \mathcal{T}_{\theta \phi}, \mathcal{T}_{\phi \phi}, \mathcal{V}_{r}, \mathcal{V}_{\theta}, \mathcal{V}_{\phi}$ and $\mathcal{L}$ are now functions of both $\theta$ and $\phi$. In what follows, $\mathcal{P}=\frac{1}{3}\left(\mathcal{T}_{r r}+\mathcal{T}_{\theta \theta}+\mathcal{T}_{\phi \phi}\right)$ is the average stress, while $\mathcal{P}^{-1}=\frac{1}{3}\left(\left(\mathcal{T}^{-1}\right)_{r r}+\left(\mathcal{T}^{-1}\right)_{\theta \theta}+\left(\mathcal{T}^{-1}\right)_{\phi \phi}\right)$ with $\left(\mathcal{T}^{-1}\right)_{i j}$ referring to the $i j$-entry of the tensor $\mathcal{T}^{-1}$ (note that $\mathcal{P}^{-1} \neq 1 / \mathcal{P}$ ).

\section{von Mises model}

$$
\begin{aligned}
\partial_{\theta} \mathcal{T}_{r \theta}+\csc (\theta) \partial_{\phi} \mathcal{T}_{r \phi}+3 \mathcal{T}_{r r}-\mathcal{T}_{\theta \theta}-\mathcal{T}_{\phi \phi}+\cot (\theta) \mathcal{T}_{r \theta} & =-\rho g \cos (\theta) \\
\partial_{\theta} \mathcal{T}_{\theta \theta}+\csc (\theta) \partial_{\phi} \mathcal{T}_{\theta \phi}+4 \mathcal{T}_{r \theta}+\cot (\theta)\left(\mathcal{T}_{\theta \theta}-\mathcal{T}_{\phi \phi}\right) & =\rho g \sin (\theta) \\
\partial_{\theta} \mathcal{T}_{\theta \phi}+\csc (\theta) \partial_{\phi} \mathcal{T}_{\phi \phi}+4 \mathcal{T}_{r \phi}+2 \cot (\theta) \mathcal{T}_{\theta \phi} & =0 \\
2 \mathcal{V}_{r} & =\mathcal{L}\left(\mathcal{T}_{r r}-\mathcal{P}\right) \\
-\frac{1}{2}\left(\partial_{\theta} \mathcal{V}_{r}-3 \mathcal{V}_{\theta}\right) & =\mathcal{L} \mathcal{T}_{r \theta} \\
-\partial_{\theta} \mathcal{V}_{\theta}-\mathcal{V}_{r} & =\mathcal{L}\left(\mathcal{T}_{\theta \theta}-\mathcal{P}\right) \\
-\frac{1}{2}\left(\csc (\theta) \partial_{\phi} \mathcal{V}_{r}-3 \mathcal{V}_{\phi}\right) & =\mathcal{L} \mathcal{T}_{r \phi} \\
-\frac{1}{2}\left(\partial_{\theta} \mathcal{V}_{\phi}+\csc (\theta) \partial_{\phi} \mathcal{V}_{\theta}-\cot (\theta) \mathcal{V}_{\phi}\right) & =\mathcal{L} \mathcal{T}_{\theta \phi} \\
-\left(\csc (\theta) \partial_{\phi} \mathcal{V}_{\phi}+\mathcal{V}_{r}+\cot (\theta) \mathcal{V}_{\theta}\right) & =\mathcal{L}\left(\mathcal{T}_{\phi \phi}-\mathcal{P}\right) \\
|\operatorname{dev} \mathcal{T}|^{2} & =2 s^{2} \mathcal{P}^{2}
\end{aligned}
$$

\section{Matsuoka-Nakai model}

$$
\begin{aligned}
\partial_{\theta} \mathcal{T}_{r \theta}+\csc (\theta) \partial_{\phi} \mathcal{T}_{r \phi}+3 \mathcal{T}_{r r}-\mathcal{T}_{\theta \theta}-\mathcal{T}_{\phi \phi}+\cot (\theta) \mathcal{T}_{r \theta} & =-\rho g \cos (\theta), \\
\partial_{\theta} \mathcal{T}_{\theta \theta}+\csc (\theta) \partial_{\phi} \mathcal{T}_{\theta \phi}+4 \mathcal{T}_{r \theta}+\cot (\theta)\left(\mathcal{T}_{\theta \theta}-\mathcal{T}_{\phi \phi}\right) & =\rho g \sin (\theta), \\
\partial_{\theta} \mathcal{T}_{\theta \phi}+\csc (\theta) \partial_{\phi} \mathcal{T}_{\phi \phi}+4 \mathcal{T}_{r \phi}+2 \cot (\theta) \mathcal{T}_{\theta \phi} & =0 \\
2 \mathcal{V}_{r} & =-\mathcal{L}\left(\left(\mathcal{T}^{-1}\right)_{r r}-\mathcal{P}^{-1}\right), \\
-\frac{1}{2}\left(\partial_{\theta} \mathcal{V}_{r}-3 \mathcal{V}_{\theta}\right) & =-\mathcal{L}\left(\mathcal{T}^{-1}\right)_{r \theta}, \\
-\partial_{\theta} \mathcal{V}_{\theta}-\mathcal{V}_{r} & =-\mathcal{L}\left(\left(\mathcal{T}^{-1}\right)_{\theta \theta}-\mathcal{P}^{-1}\right), \\
-\frac{1}{2}\left(\csc (\theta) \partial_{\phi} \mathcal{V}_{r}-3 \mathcal{V}_{\phi}\right) & =-\mathcal{L}\left(\mathcal{T}^{-1}\right)_{r \phi}
\end{aligned}
$$




$$
\begin{aligned}
-\frac{1}{2}\left(\partial_{\theta} \mathcal{V}_{\phi}+\csc (\theta) \partial_{\phi} \mathcal{V}_{\theta}-\cot (\theta) \mathcal{V}_{\phi}\right) & =-\mathcal{L}\left(\mathcal{T}^{-1}\right)_{\theta \phi}, \\
-\left(\csc (\theta) \partial_{\phi} \mathcal{V}_{\phi}+\mathcal{V}_{r}+\cot (\theta) \mathcal{V}_{\theta}\right) & =-\mathcal{L}\left(\left(\mathcal{T}^{-1}\right)_{\phi \phi}-\mathcal{P}^{-1}\right), \\
\left(\mathcal{T}_{r r}+\mathcal{T}_{\theta \theta}+\mathcal{T}_{\phi \phi}\right)\left(\left(\mathcal{T}^{-1}\right)_{r r}+\left(\mathcal{T}^{-1}\right)_{\theta \theta}+\left(\mathcal{T}^{-1}\right)_{\phi \phi}\right) & =9\left(1+\mu^{2}\right)
\end{aligned}
$$

The above equations have to be satisfied on the spherical cap $\{(\theta, \phi) ;-\pi<$ $\phi<\pi, 0<\theta<\mathcal{C}(\phi)\}$. The boundary conditions $(8,9)$ can be expressed in terms of the above unknowns. The unit interior normal vector $N$ on the outside wall $\theta=\mathcal{C}(\phi)$ takes the form

$$
N=\left[N_{r}, N_{\theta}, N_{\phi}\right]=\left[0,-\sin \theta, \mathcal{C}^{\prime}(\phi)\right] / \sqrt{\sin ^{2} \theta+\mathcal{C}^{\prime}(\phi)^{2}} .
$$

Therefore, when $\theta=\mathcal{C}(\phi), 0<\phi<2 \pi$, one has

$$
\begin{gathered}
\mathcal{V}_{\theta} N_{\theta}+\mathcal{V}_{\phi} N_{\phi}=0 \\
\mathcal{V}_{\phi}\left(\mathcal{T}_{r \theta} N_{\theta}+\mathcal{T}_{r \phi} N_{\phi}\right)-\mathcal{V}_{r}\left[-\mathcal{T}_{\theta \theta} N_{\theta}^{2} N_{\phi}+\mathcal{T}_{\theta \phi} N_{\theta}\left(1-2 N_{\phi}^{2}\right)+\mathcal{T}_{\phi \phi} N_{\phi} N_{\theta}^{2}\right]=0 \\
\sqrt{\left(\mathcal{T}_{r \theta} N_{\theta}+\mathcal{T}_{r \phi} N_{\phi}\right)^{2}+\left(\mathcal{T}_{\theta \theta} N_{\theta} N_{\phi}-\mathcal{T}_{\phi \phi} N_{\theta} N_{\phi}+\mathcal{T}_{\theta \phi}\left(N_{\phi}^{2}-N_{\theta}^{2}\right)\right)^{2}} \\
=\mu_{w}\left(\mathcal{T}_{\theta \theta} N_{\theta}^{2}+2 \mathcal{T}_{\theta \phi} N_{\theta} N_{\phi}+\mathcal{T}_{\phi \phi} N_{\phi}^{2}\right)
\end{gathered}
$$

where (37) corresponds to condition (8). The vectorial condition (9) is rewritten into two scalar conditions. First, we impose $\tau_{T} \times v=0$ on the wall; the cross product of two two-dimensional vectors being a scalar, this leads to (38). Second, $\left|\tau_{T}\right|=\mu_{w} \tau_{N}$ holds, leading to (39). From Section 2, the stress tensor $T$ is positive definite; consequently, the right hand of (39) is positive since $T_{N}=N^{t} T N>0^{5}$. The above set of equations defines the unknowns $\mathcal{V}_{r}, \mathcal{V}_{\theta}$, $\mathcal{V}_{\phi}$ and $\mathcal{L}$ only up to a positive multiplicative constant. To eliminate this indeterminacy, the value of one component of the velocity, say $\mathcal{V}_{r}$, is fixed at one point in the computational domain. Here, we choose (see also (19))

$$
\mathcal{V}_{r}\left(\Theta_{0}, \Phi_{0}\right)=v^{\star}<0
$$

where $\Theta_{0}$ and $\Phi_{0}$ are defined below and where $v^{\star}$ in effect scales the flow rate out of the hopper. Note that no conditions are needed at $\theta=0$ (or more

5 Conditions $(38,39)$ are invariant under a sign change of $\tau_{T}$. Clearly, only the sign given in (9) is physical. The numerical results given below have been checked to correspond to physical solutions: the correct sign in (39) is taken, the computed stress tensor is positive definite and the plasticity factor $\mathcal{L}$ is positive. This sign indeterminacy does not exist in the purely radial case where (39) simplifies into (18). For the above full problem, physical radial solutions are used as initial solutions, see [4] for more details. 
precisely, considering only nonsingular solutions at that point is a boundary condition). Further, $2 \pi$-periodicity is imposed in the $\phi$-direction.

Along with the above unknowns, a stream function $\Psi$ is computed to illustrate the appearance of secondary circulation in the velocity field. Under assumption (10), the incompressibility condition $\operatorname{div} v=0$ reads here

$$
\partial_{\theta}\left(\sin \theta \mathcal{V}_{\theta}\right)+\partial_{\phi} \mathcal{V}_{\phi}=0
$$

A stream function $\Psi$ can then be introduced through

$$
\partial_{\phi} \Psi=\sin \theta \mathcal{V}_{\theta} \quad \text { and } \quad \partial_{\theta} \Psi=-\mathcal{V}_{\phi}
$$

\section{$5 \quad$ Numerical results}

The discretization used to solve the above system is identical to the one used in [4] and is only briefly described here. In order to simplify the numerics, the problem is mapped onto a rectangular computational domain. We define the new coordinates

$$
\Theta=\theta_{w} \frac{\theta}{\mathcal{C}(\phi)} \quad \text { and } \quad \Phi=\phi
$$

The computational domain is now simply $\left(0, \theta_{w}\right] \times\left[-\phi_{w}, \phi_{w}\right)$, where $\phi_{w}$ corresponds to the smallest interval of periodicity of the solution in the $\phi$-direction.

The equations (20-39) are written in the new coordinate system. The resulting problem can be discretized by collocation; Chebyshev collocation at the Chebyshev-Gauss-Radau points is used in $\Theta$, while Fourier-cosine collocation at the Fourier collocation points is used in $\Phi$. More precisely, the numerical solution takes the form

$$
U_{N M}(\Theta, \Phi)=\sum_{n=0}^{N-1} \sum_{m=-M / 2}^{M / 2-1} U_{n m} \psi_{n}(\Theta) e^{i m \pi\left(\frac{\Phi}{\phi_{w}}+1\right)}
$$

where $\left\{\psi_{n}\right\}_{n=0}^{N-1}$ are the Lagrange interpolation polynomials at the ChebyshevGauss-Radau nodes on $\left(0, \theta_{w}\right]$.

To illustrate the differences between the two plasticity models, we consider two families of pyramidal domains which approximate industrial hoppers with square and oblong cross sections respectively. The former family is described by 

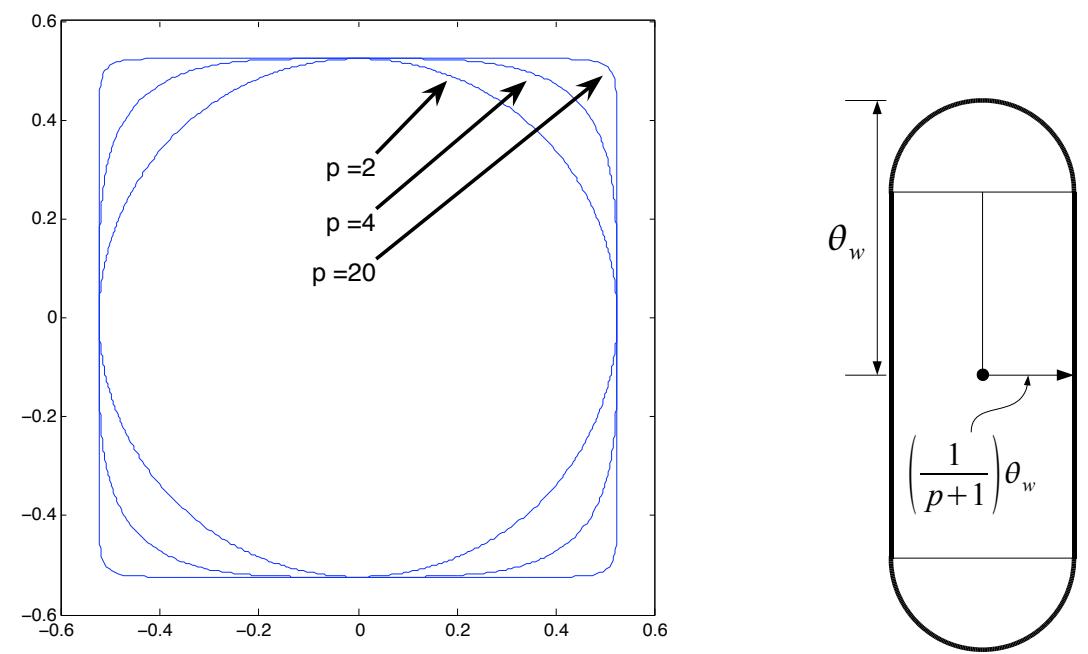

Fig. 2. Left: cross section of the "square" hopper (43) for $p=2,4,20$ ( $p=20$ and $\theta_{w}=30^{\circ}$ in the calculations below); right: cross section of the oblong, nozzle-shaped hopper ( $p=2$ and $\theta_{w}=30^{\circ}$ in the calculations below).

$$
\mathcal{C}(\phi)=\frac{\theta_{w}}{\left(\cos ^{p} \phi+\sin ^{p} \phi\right)^{1 / p}}, \quad 2 \leq p<\infty,
$$

see Figure 2, left. These domains are similar to the unit ball in the $p$-norm. In the calculations below, $p$ was taken as 20 and $\theta_{w}$ is 30 degrees.

The second family is best described geometrically, see Figure 2, right.

\subsection{Explanation of Figures}

Figures 3 and 5 compare the two different plasticity models in the two above geometries, having evaluated all quantities on a horizontal plane normal to the $z$-axis. In each row, von Mises is on the left and Matsuoka-Nakai on the right. The first row shows the average stresses, $\mathcal{P}$. The second row presents the vertical component of the velocity, i.e., $\mathcal{V}_{z}=\mathcal{V} \cdot e_{z}$, scaled such that $\mathcal{V}_{z}$ attains a minimum of -1 (i.e. a maximum in magnitude, in the negative $z$ direction). In the third row, color represents speed in the horizontal plane, i.e., $\left|\mathcal{V}-\mathcal{V} \cdot e_{z} e_{z}\right|$ and black curves are streamlines (i.e., level lines of the stream function $\Psi$ ) delineating areas of secondary circulation.

\subsection{Results for Square Hoppers}

Figure 3 makes apparent the differences between the two constitutive laws in the square hopper. While the stress fields for Matsuoka-Nakai and von Mises are similar, the former displays stronger corner effects. Not only does 

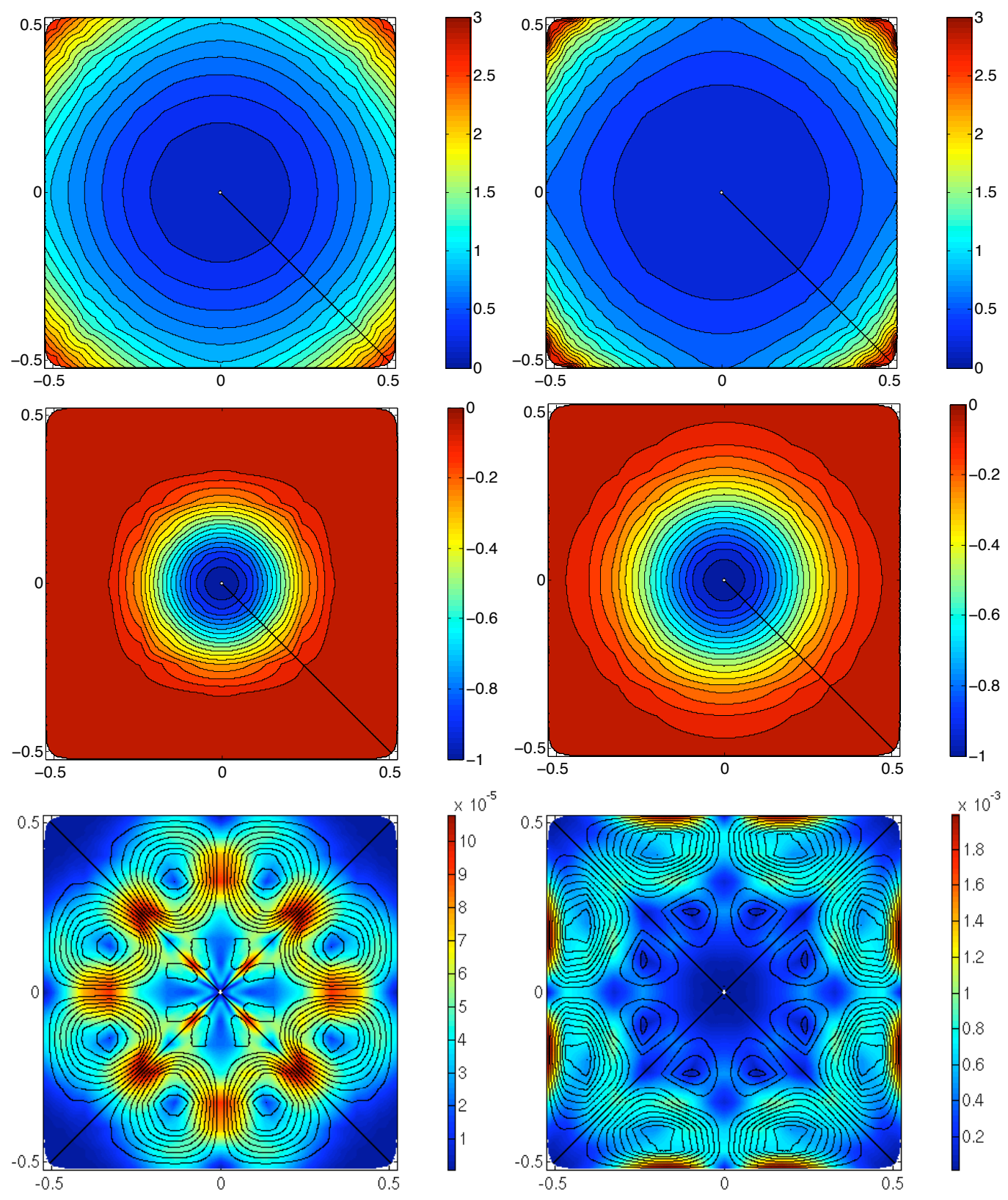

Fig. 3. Flow in a vertical square hopper (43) with $p=20$ and $\theta_{w}=30^{\circ}$; material parameters are: angle of internal friction $\delta=30^{\circ}$, coefficient of wall friction $\mu_{w}=0.3$. Left pictures: von Mises; right pictures: Matsuoka-Nakai. From top to bottom: average stress $\mathcal{P}=\frac{1}{3}\left(\mathcal{T}_{r r}+\mathcal{T}_{\theta \theta}+\mathcal{T}_{\phi \phi}\right)$, vertical component of velocity $\mathcal{V}_{z}$ and horizontal speed/streamlines (see text for details).

Matsuoka-Nakai achieve a higher stress in the corners, but the gradient of the stress field is also higher in those regions.

A striking difference in the square hopper is that von Mises predicts a flow which appears much nearer to the mass flow limit, the point at which some portion of the material ceases to deform [8]. Under the von Mises plasticity law, 

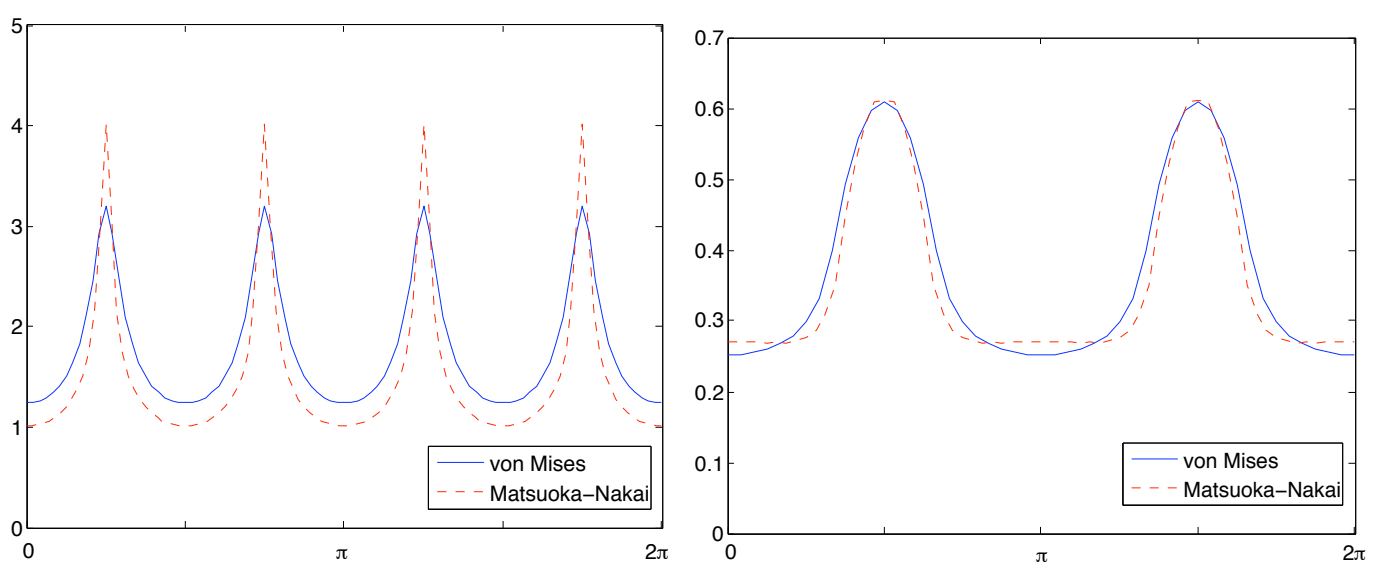

Fig. 4. Normal stress on hopper walls. Square hopper, left; oblong hopper; right. Material parameters: angle of internal friction $\delta=30^{\circ}$, half opening angle $\theta_{w}=30^{\circ}$, coefficient of wall friction $\mu_{w}=0.3$.

the bulk of the material nearest to the walls is moving downward slowly and nearly uniformly while a roughly circular channel in the center is moving and deforming very rapidly. There are similar slow regions near the walls under the Matsuoka-Nakai plasticity law, but the appearance of a fast-moving channel in the center is less pronounced.

Another important distinction between the two models in the square hopper is that the secondary circulation is an order of magnitude stronger for Matsuoka-Nakai than for von Mises. The third row in Figure 3 demonstrates this difference by displaying the horizontal speed $\left(\left|\mathcal{V}-\mathcal{V} \cdot e_{z} e_{z}\right|\right)$. Preliminary comparison with laboratory experiments shows uncanny agreement with the Matsuoka-Nakai prediction, and casts doubt on the accuracy of the von Mises prediction [11]. A more detailed comparison between the models and experimental results will be pursued in a forthcoming work [1].

The representation of speed in the third row of Figure 3 also reveals the effect of the fast-moving center channel in the von Mises case. This channel pulls material in from the sides inducing the fastest horizontal speed on the hopper's interior; for Matsuoka-Nakai, the fastest speed is at the wall where material is being pulled from near the corners toward the steepest point along the hopper wall.

The third row of Figure 3 also shows the location of circulation cells as black streamlines. Grains along a wall move from the corner toward the midpoint on that wall, where the wall is steepest. There are also circulation cells wholly in the interior of the hopper. In both models, however, the secondary circulation is a small effect, from 2 to 4 orders of magnitude smaller than the vertical component of velocity. 
Another effect of the loss of axisymmetry can be plainly seen in the normal stress at the wall. In Figure 4, left, the normal stress along the circumference of the square hopper is computed for both models. For both models, the maximum normal stress is found at the corners. Further, those corner effects are more pronounced for Matsuoka-Nakai than they are for von Mises. It should also be noted that this kind of predictions is fully outside the range of typical radial models such as Jenike's. Accurate predictions of the wall stresses are fundamental for the study of silo failures. Such a study could be performed by coupling the current results with shell stress modeling codes, reinforcing the importance of the present approach.

\subsection{Results for Oblong Hoppers}

Results showing the same data, albeit in an oblong hopper geometry, are shown in Figure 5. In the first row of graphs, the general shape of the average stress fields is similar for both models, with the maximum average stress along the wall at the narrow end of the hopper and the minimum along the steeper flat wall. However, along the flat walls, the Matsuoka-Nakai plasticity law predicts an almost uniform average stress while the von Mises law predicts an average stress with more variation.

This is also reflected in the normal stress, seen in Figure 4, right, where the maximum and minimum values of the normal stress are nearly identical between the two models, but the Matsuoka-Nakai model has a more uniform normal stress along the flat walls of the hopper.

Returning to Figure 5, the second row of graphs again represents the vertical component of velocity. Unlike the square hopper, both plasticity laws make similar predictions, both in magnitude and in the shape of the field.

The third row of graphs in Figure 5 shows that, again, the circulation characteristics predicted by the two models differ substantially. While the circulation cells themselves (demarcated by black curves) are not dissimilar, the speed of the circulation (indicated by color) predicted by the Matsuoka-Nakai model is an order of magnitude larger than that of the von Mises model. Furthermore, for the von Mises plasticity law, there are two regions of grains internal to the hopper which are moving about as rapidly as grains move along the steep, flat wall. This effect is not nearly so strong for the Matsuoka-Nakai plasticity law. 

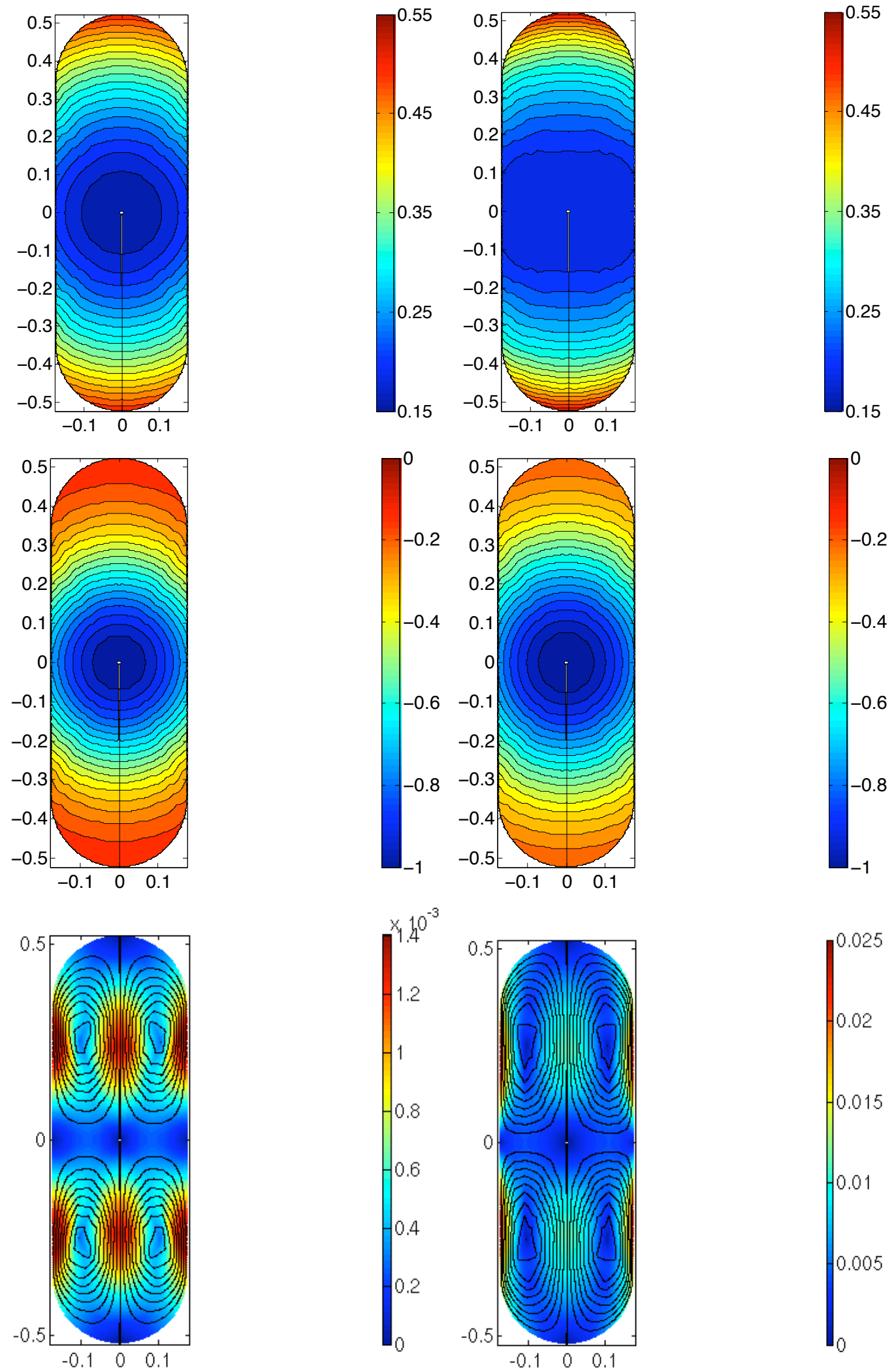

Fig. 5. Flow in a vertical nozzle shaped hopper (see Figure 2); material parameters are: angle of internal friction $\delta=30^{\circ}$, coefficient of wall friction $\mu_{w}=0.3$. Left pictures: von Mises; right pictures: Matsuoka-Nakai. From top to bottom: average stress $\mathcal{P}$, vertical component of velocity $\mathcal{V}_{z}$ and horizontal speed/streamlines (see text for details). 


\section{Conclusions}

The construction of similarity solutions corresponding to granular flows in nonaxisymmetric hoppers was started in $[4,5]$; it is extended here to MatsuokaNakai materials. While Jenike's original method is limited to conical hoppers (right circular cones), the present approach can be applied to pyramidal (selfsimilar) hoppers of arbitrary cross section that are common in industrial applications. It should be noted however that the case of general fully three dimensional containers is outside the scope of the present contribution and, to the authors' knowledge, is still a largely open problem (in part due to the difficulty of setting up a well-posed mathematical formulation).

Phenomenological arguments leading to plasticity laws such as those considered here are poor substitutes for derivations from first principles, which regrettably are lacking in the present field. It is thus fundamental to be able to test and compare such models against one another and more importantly against experimental data. The present contribution is a necessary step in this direction. Preliminary comparisons with experiments $[1,11]$ indicate that the lesser known Matsuoka-Nakai plasticity condition leads to results that are much closer to experimental data than the widely used von Mises condition (strictly within the present context, of course). Our approach also gives access to fundamental practical information, such as the importance of corner effects on wall stresses.

\section{Acknowledgements}

The authors thank Bob Behringer and John Wambaugh for many helpful discussions and for giving them access to their experimental results.

\section{References}

[1] R.P. Behringer, P.A. Gremaud, J.V. Matthews, D.G. Schaeffer and J.F. WAMBAUGH, In preparation.

[2] R.I. BorJa, K.M. SAma And P.F. SAnZ, On the numerical integration of three-invariant elastoplastic constitutive models, Comp. Methods Appl. Mech. Engrg., 192 (2003), pp. 1227-1258.

[3] I.F. Collins, A systematic procedure for constructing critical state models in three dimensions, Int. J. Solids and Structures, 40 (2003), pp. 4379-4397. 
[4] P.A. Gremaud, J.V. Matthews and M. O'Malley, On the Computation of Steady Hopper Flows II: von Mises Materials in Various Geometries, J. Comput. Phys., (200) 2004, pp. 639-653,

[5] P.A. Gremaud, J.V. Matthews and D.G. Schaeffer, Secondary circulation in granular flow through nonaxisymmetric hoppers, SIAM J. App. Math., (64) 2003, pp. 583-600.

[6] A.W. Jenike, Gravity flow of bluk solids, Bulletin No. 108, Utah Eng. Expt. Station, University of Utah, Salt Lake City (1961).

[7] H. Matsuoka and T. Nakai, Stress-deformation and strength characteristics of soils under three different principal stresses in Proc. JSCE, No 232, 1974, pp. 59-70.

[8] R.M. Nedderman, Static and kinematic of granular materials, Cambridge University Press, 1992.

[9] M.J.D. Powell, A Fortran Subroutine for Solving Systems of Nonlinear Algebraic Equations, Numerical Methods for Nonlinear Algebraic Equations, (P. Rabinowitz, ed.), Ch.7, 1970.

[10] D.G. Schaeffer, Instability in the evolution equations describing incompressible granular flow, J. Diff. Eq., 66 (1987), pp. 19-50.

[11] J.F. Wambaugh, R.P. Behringer, Asymmetry-induced circulation in granular hopper flows, Powders and Grains 2005, (R. Garcia-Rojo, H.J. Herrmann, S. McNamara, eds.), A.A. Balkema, 2005, pp.915-918. 